\title{
CREUTZFELDT-JAKOB DISEASE SURVEILLANCE IN EASTERN SLOVAKIA FROM 2004 TO 2016
}

\author{
Eva Feketeová1, Dominika Jarčušková1, Alžbeta Janáková2, Eva Rozprávková3, Zlatica Cifráková4, \\ Silvia Farkašová-Inaccone ${ }^{5}$, Kvetoslava Rimárová ${ }^{6}$, Erik Dorko ${ }^{6}$, Zuzana Gdovinová ${ }^{1}$ \\ 'Department of Neurology, Faculty of Medicine, Pavol Jozef Šafárik University in Košice and Louis Pasteur University Hospital, Košice, \\ Slovak Republic \\ ${ }^{2}$ Department of Prion Diseases, Slovak Medical University in Bratislava, Bratislava, Slovak Republic \\ ${ }^{31} 1$ st Private Hospital Košice-Šaca, Košice, Slovak Republic \\ ${ }^{4}$ Department of Neurology, Louis Pasteur University Hospital, Košice, Slovak Republic \\ ${ }^{5}$ Department of Forensic Medicine, Faculty of Medicine, Pavol Jozef Šafárik University in Košice, Košice, Slovak Republic \\ ${ }^{6}$ Department of Public Health and Hygiene, Faculty of Medicine, Pavol Jozef Šafárik University in Košice, Slovak Republic
}

\section{SUMMARY}

Objectives: An extraordinary incidence of genetic Creutzfeldt-Jakob disease (GCJD) appearing in clusters in the Slovak Republic was described in the 1990's. The aim of the study was to analyse data of CJD cases obtained from surveillance in Eastern Slovakia (ES) (2004-2016), the region outside the described geographical clusters.

Methods: The database set in the project was the source for epidemiological and clinical analysis of CJD cases.

Results: The incidence of CJD in ES (2004-2016) was 1.7/million person-years (95\% Cl 1-2.4); the incidence increase in the last five years (2012-2016) was comparable to the whole country. Twenty seven of 29 reported CJD cases were available for analysis (mean age 59 years, F/M 15/12). The proportion of gCJD (E200K mutation) cases remained dominant (78\%), with 9 familiar cases originating in 4 families. Analysis of the clinical features revealed shorter duration of the symptomatic phase in sporadic CJD (SCJD) (3.4 months) versus gCJD (5.15 months). Cognitive/ behavioural changes, insomnia, and sensory disturbance were more pronounced in the early symptoms of gCJD. Periodic EEG discharges were more frequent in SCJD (83\%) than gCJD (56\%), all 19 available MR findings were CJD specific and localisation of abnormalities varied amongst the CJD forms.

Conclusions: The surveillance of CJD in ES (2004-2016) showed an increased incidence of CJD in ES, reaching the incidence rate of the whole country, with a permanent proportion of $70 \% \mathrm{gCJD}$ cases based on the E200K mutation. Clinical, electrophysiological and MR features of SCJD and gCJD cases were in conformity with already published data. Epidemiological analysis of CJD in ES shows increasing detection of CJD but also suggests that current routine surveillance systems for CJD may underestimate the true burden of disease, especially sporadic cases in Slovakia.

Key words: Creutzfeldt-Jakob disease, epidemiology, E200K mutation, genetic CJD, sporadic CJD

Address for correspondence: E. Feketeová, Department of Neurology, Faculty of Medicine, Pavol Jozef Šafárik University in Košice and Louis Pasteur University Hospital, Trieda SNP 1, 04011 Košice, Slovak Republic. E-mail: eva.feketeova@upjs.sk

https://doi.org/10.21101/cejph.a5277

\section{INTRODUCTION}

Creutzfeldt-Jakob disease (CJD) is a rare fatal neurodegenerative disorder from a family of transmissible spongiform encephalopathies. The incidence of CJD varies between 0.5 and 1.5 cases per million persons per year with no gender predisposition and a mean age of $65+$ at the onset of disease with a range of 14 to 92 years (1).

CJD is a prion disease caused by the accumulation of the pathological prion protein in the central nervous system. Prions are composed of abnormal isoforms of a host-encoded glycoprotein, a prion protein. Prion propagation involves recruitment of host cellular prion protein (primarily of $\alpha$-helical structure) into a disease specific isoform rich in $\beta$-sheet structure. About $85 \%$ of CJD cases occur sporadically (sporadic CJD - sCJD), without clear aetiology $(2,3)$. Less frequent genetic CJD (gCJD) cases occur based on mutation of prion protein gen (PRNP). Individual PRNP mutations show variable geographical distribution, frequency and gene penetrance. A genetic cluster of gCJD with the unique presence of E200K mutation was detected in Slovakia $(1,4,5)$. Genetic testing in Slovakia identified familial clusters of the disease in northern Slovakia with a gene penetrance in the affected families of about $60 \%$, raising the probability of environmental cofactors playing a role in disease manifestation in separated siblings (6-8).

In the presented study we set up the database of CJD in eastern Slovakia (ES) and analysed the CJD cases to see if improved diagnostic methods and increasing illness awareness have resulted in the increasing number of diagnosed CJD cases, if there are regional differences in Slovakia, if there is a persistent dominant proportion of $\mathrm{gCJD}$ and what are the clinical, electrophysiological and MR features in the Slovak sample of CJD within the period of 2004-2016. 


\section{MATERIALS AND METHODS}

Twenty-nine CJD cases of probable and definite CJD, coming from ES, in the 2004-2016 period were identified. To be included into the database the patients had to be resident for a minimum 2/3 of their life-span in ES.

The diagnosis of probable sCJD ( 2 cases) was based on rapidly evolving dementia ( $<2$ years), presence of periodic sharp wave complexes (PSWC) with triphasic morphology in EEG recordings and/or presence of 14-3-3 protein in cerebrospinal fluid examination and at least two of the following 4 clinical signs: myoclonus, ataxia and/or visual signs and symptoms, extrapyramidal and/ or pyramidal signs and symptoms, and akinetic mutism. Definite SCJD was confirmed by histopathology as the presence of spongiform degeneration and gliosis and/or Western blot presence of protease-resistant PrP.

Diagnosis of genetic prion disease required a combination of clinical features (dementia, psychiatric symptoms, ataxia, dysartria, myoclonus, pyramidal signs, chorea, seizures, autonomic disturbances), neuropathological findings (spongiform degeneration and astrogliosis diffusely distributed throughout the cortex and deep nuclei of the brain), and a PRNP pathogenic variant (E200K).

Peripheral blood was tested for the E200K mutation (no other gene mutations were tested) and the distribution of the polymorphism M129V form. A 14-3-3 protein in cerebrospinal fluid (CSF) was evaluated by Western Blot method. The presence of pathological prion proteins in autopsy samples of the brain was examined immunohistochemically and neurohistopathologically. Examinations of the 14-3-3 protein, molecular biological analysis and confirmation of pathological prion proteins in the brain were performed at the Department of Prion Diseases in Bratislava.

Clinical data were collected with respect to age of onset of the symptoms, gender, family history, the spectrum of onset symptoms, duration of symptomatic phase, presence of CJD or other dementia in siblings. Electroencephalographic (EEG) and magnetic resonance (MR) findings in T2, FLAIR, and DWI imaging were evaluated. Negative files, especially older ones, were re-evaluated by an experienced neurologist.

All the data were compared for sporadic and genetic CJD cases by descriptive statistics methods.

\section{RESULTS}

Twenty nine CJD cases identified from 2004 to 2016 in an ES population of 1.617,347 (according to the Slovak Statistical Office as of 31. 12. 2015) made the incidence of CJD in ES 1.7/ million person-years (95\% CI 1-2.4). The incidence of CJD differed during this period; the incidence in the first five years of the observed period (2004-2008) was $1 /$ million person-years (95\% CI 0.07-0.13), significantly lower than in the second period (2012-2016) which was 2.7/million person-years (95\% CI 1.6-3.9). Furthermore, the years 2012-2016 did not differ in incidence for ES and the whole country (3.2/million person-years, 95\% CI 2.2-4.2), as shown in Figure 1. There was no gender predisposition (13:16) and the mean age was 60 years. In 5 cases (17\%) histopathological examination of the brains was not performed ( 3 cases of probable CJD without the E200K mutation and 2 cases with the presence of E200K mutation).

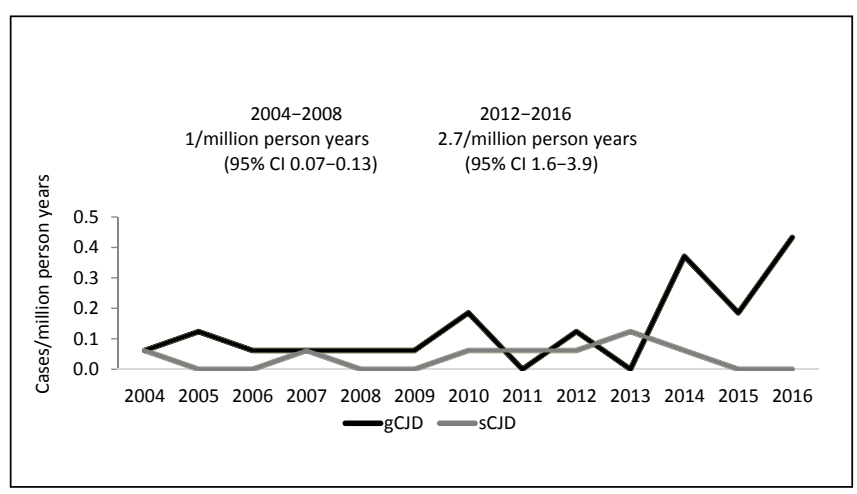

Fig. 1. Incidence of Creutzfeldt-Jakob Disease (CJD) in Eastern Slovakia in 2004-2016.

sCJD - sporadic Creutzfeldt-Jakob Disease; gCJD - genetic Creutzfeldt-Jakob Disease

Table 1. Demographic and genetic features of CreutzfeldtJakob Disease (CJD) cases $(N=27)$

\begin{tabular}{|l|c|c|c|}
\hline & CJD & SCJD & gCJD (+E200K) \\
\hline Patients (n) & 27 & 6 & 21 \\
\hline Mean age \pm SD & $58.9 \pm 7.6$ & $61.8 \pm 9.6$ & $58.9 \pm 7.4$ \\
\hline Females/Males & $15 / 12$ & $3 / 3$ & $12 / 9$ \\
\hline Familial cases & $9(33 \%)$ & & $9(43 \%)$ \\
\hline $\begin{array}{l}\text { Symptomatic } \\
\text { phase duration } \\
\text { (months) }\end{array}$ & 4.8 & 3.4 & 5.15 \\
\hline M129 V polymorphism & & \\
\hline Met/Met & $19(73 \%)$ & $4(80 \%)$ & $15(71 \%)$ \\
\hline Met/Val & $6(23 \%)$ & & $6(29 \%)$ \\
\hline Val/Nal & $1(4 \%)$ & $1(20 \%)$ & \\
\hline Protein 14-3-3 & $19(73 \%)$ & $4(67 \%)$ & $15(71 \%)$ \\
\hline
\end{tabular}

SCJD - sporadic CJD; gCJD - genetic CJD with the presence of E200K mutation

Two cases out of 29 were excluded from the final analysis because their medical records were not found ( $1 \mathrm{gCJD}$ case from 2008, 1 sCJD case from 2013). The mean age of 27 cases with available records was 59 years, female/male ratio was $15 / 12$.

The results of molecular analysis stratified $6(22 \%)$ patients to sCJD and $21(78 \%)$ to gCJD. Four families were identified in 21 gCJD cases with 9 affected siblings together: $38 \%$ of gCJD were familial cases. Examination of M129V polymorphism revealed Met/Met polymorphism predominance in both SCJD and gCJD (Table 1).

A mean duration of symptomatic phase of CJD was 4.8 months, shorter in SCJD: 3.4 months. Thirty-seven percent of CJD cases manifested with cognitive and behavioural changes. Gait disturbance and ataxia were present in one third of cases whereas extrapyramidal signs and myoclonus were present in one fourth of cases. Epileptic seizures and pyramidal tract lesions were not found in the early clinical signs. Figure 2 shows the clinical features in comparison between SCJD and gCJD in the onset of CJD.

With the progression of the disorder (usually at the time when the patients were admitted to hospital) the majority of patients 


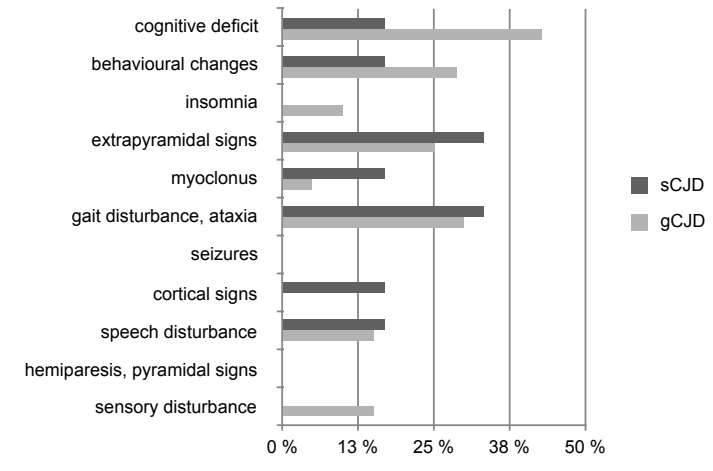

Fig. 2. Clinical features of sporadic Creutzfeldt-Jakob Disease and genetic Creutzfeldt-Jakob Disease - early signs of the disorder.

sCJD - sporadic Creutzfeldt-Jakob Disease; gCJD - genetic Creutzfeldt-Jakob Disease

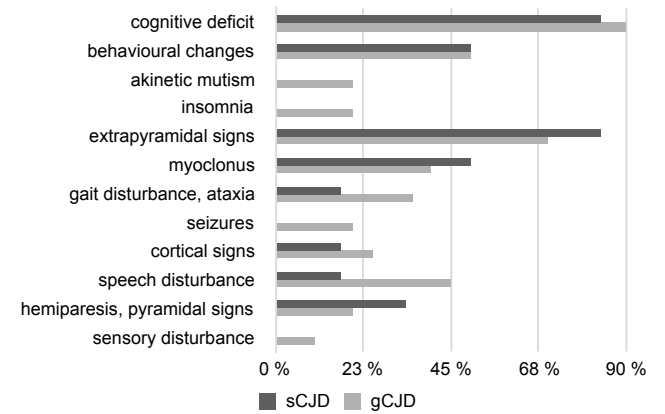

Fig. 3. Clinical features of $s C J D$ and gCJD in fully manifested stage of the disorder.

sCJD - sporadic Creutzfeldt-Jakob Disease; gCJD - genetic Creutzfeldt-Jakob Disease

developed cognitive deficit ( $89 \%$ ), behaviour disturbance (52\%), and changed level of consciousness $(70 \%)$. Seventy percent showed extrapyramidal signs, $44 \%$ myoclonus, and $33 \%$ ataxia. Seizures and other cortical and pyramidal signs were noted in one fifth of patients. Figure 3 shows a comparison of the features in SCJD and gCJD cases in the stage of fully manifested clinical symptoms.

Electrophysiological examination yielded the presence of PSWC in $83 \%$ sCJD and $56 \%$ gCJD. Thirty-three percent of sCJD cases were lateralised PSWC ( $5 \%$ in gCJD). Forty two percent of gCJD cases had nonspecific abnormal or normal EEG findings (Figure 4). MR findings were available in 19 out of 27 cases ( 6 were not available for re-evaluation or were not done in $\mathrm{gCJD}, 2$ in SCJD). SCJD showed abnormal MR findings in basal ganglia in all of the cases and in the cortex in $25 \%$. Abnormal MR findings in basal ganglia (69\%), cortex $(56 \%)$, thalamus $(19 \%)$ were present in gCJD cases, showing dissemination of abnormalities, and lateralised findings. All 19 MR examinations were CJD specific (6 MRs were considered false negative after re-evaluation, the scans came from years prior to 2010) (Figure 5).

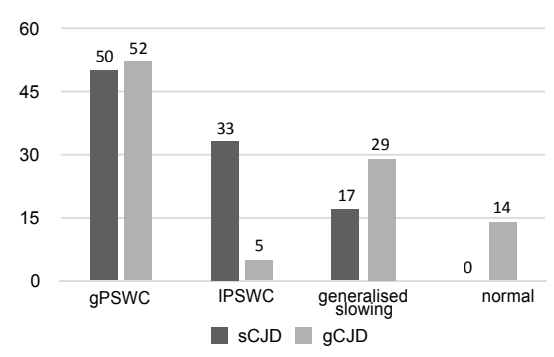

Fig. 4. EEG findings in sporadic Creutzfeldt-Jakob Disease and genetic Creutzfeldt-Jakob Disease cases.

sCJD - sporadic Creutzfeldt-Jakob Disease; gCJD - genetic Creutzfeldt-Jakob Disease; gPSWC - Generalised Periodic Sharp Wave Complexes; IPSWC - Lateralised Periodic Sharp Wave Complexes

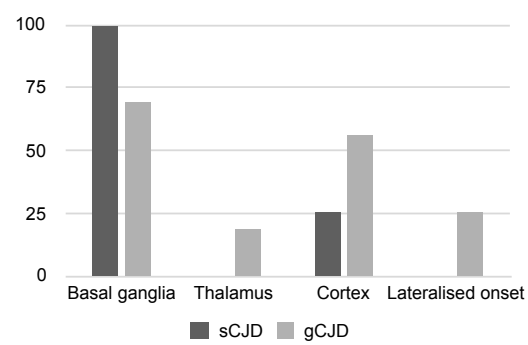

Fig. 5. MR findings in $s C J D$ and gCJD cases - localisation of the pathological signal on T2 weighted and DWI sequences. sCJD - sporadic Creutzfeldt-Jakob Disease; gCJD - genetic Creutzfeldt-Jakob Disease

\section{DISCUSSION}

The incidence of the CJD in ES from 2004 to 2016 was $1.7 /$ million person-years ( $95 \%$ CI 1-2.4), showing an incidence increase and reaching the incidence rate of the whole country in 2012-2016. The genetic form constituted 78\% of the cases and $38 \%$ of $\mathrm{gCJD}$ were familial cases. There was no gender predisposition. Twenty seven of $29 \mathrm{CJD}$ cases were available for analysis (mean age 59 years, F/M 15/12). Genetic analysis of gCJD cases revealed the presence of E200K mutation (100\%). At the time of fully expressed clinical signs all the patients showed cognitive deficit and/or behavioural changes and/or changes in level of consciousness. Patients with gCJD suffered from insomnia, seizures and sensory disturbance, while those with sCJD did not. In the onset symptoms, patients with gCJD showed earlier cognitive deficit and/or behavioural changes, while patients with SCJD experienced extrapyramidal signs, ataxia and cortical symptoms. Objective cognitive testing was not available in patients with speech disturbance or those with progressed clinical deterioration on the level of akinetic mutism. This fact explains the surprising number of patients who did not have dementia listed among 
their recorded clinical symptoms even during the fully clinically expressed stage of the disease. All MR findings in SCJD patients revealed specific abnormalities, $83 \%$ of EEG results were specifically abnormal. Disease specific findings on MR and EEG were less frequent in gCJD cases.

Slovakia has been participating in the European network Euro CJD since 1993 with six other European countries (Austria, Germany, France, Italy, Nederland, and UK) secondarily expanded to other European and non European countries such as Spain, Canada and Australia. Pooled data from the member states between 1999 and 2002 revealed a mortality rate 1.67 per million for all cases and 1.39 per million for sporadic CJD. Mortality rates were similar in all countries. There was heterogeneity in the distribution of cases by etiologic subtype with an excess of genetic cases in Italy and Slovakia $(9,10)$. The growth trend in Slovakia shown in the presented study did not change in following years and gCJD with the E200K mutation exceed up to $78 \%$ of cases, with an incidence in 2012-2016 of 2.2/million person-years (95\% CI $0.8-3.6)$. Tested family members in the affected families showed $35 \%$ presence of the E200K mutation with gene penetrance $59.5 \%$ in a study by Mitrova (4). There are several possible explanations for the increased incidence of CJD in recent years: systematic and more sensitive surveillance based on communication with referring neurologists, and laboratory tests with a wide spectrum of biomarkers in rapid progressive dementia have raised awareness and clinical interest in CJD. Changes in population demographics with an increase in the numbers of individuals in the age cohorts with a high incidence of CJD could participate in the increase. The overall growth of CJD incidence in ES, the region outside the described Slovak clusters, and genetic proportion of CJD do not support the role of metal environmental cofactors (8) in the incidence rates.

The observed distribution of the M129V polymorphism with a high proportion of $\mathrm{M} / \mathrm{M}$ homozygotes $(80 \%$ in $\mathrm{SCJD}, 71 \%$ in $\mathrm{gCJD})$ in the presented study indicates a considerably nonspecific CJD-risk in the general population of Slovakia.

In contrast to the increased number of gCJD cases in eastern Slovakia there is a decreasing number of SCJD cases. This fact raises the suspicion that a lot of cases have not been identified despite increasing awareness and improved diagnostic methods in Slovakia. Mitrova et al. (6) suggest that undiagnosed SCJD cases and asymptomatic "healthy" carriers of the gCJD cases left without active surveillance would further increase the risk of iatrogenic CJD. This risk is further exacerbated in a situation where there is no official database of positive tested living siblings for the transplantation registries in Slovakia.

Clinical features of E200K gCJD frequently overlap with that of SCJD although the less frequent prominent insomnia and polyneuropathy in our cohort of gCJD have also been observed by Kovacs (10). In contrast with Bertoni et al. (11) and Kovacs et al. (10) we did not find supranuclear palsy as a prominent symptom in any gCJD case in the retrospective data.

EEG has been considered a hallmark of CJD for many years. PSWC occur in EEG recordings of about two-thirds of patients with $\operatorname{SCJD}(12,13)$ as in our sample. Fulbright et al. (14) detected from brain MR the involvement of the caudate nucleus in $80 \%$ of the patients and putamen in $73 \%$ of familial CJD (fCJD) patients as consistent with our study, and the overall involvement of deep gray matter in CJD cases. Appel et al. (15) hypothesized that the finding of a high frequency of isolated deep gray matter rather than cortical involvement per MR in E200K fCJD patients may explain the low frequency of PSWC they detected, considering the presumed cortical origin of the PSWC. The involvement of the cortex in MR studies of our sample of gCJD was lower than in SCJD and could not explain the lower prevalence of PSWC in gCJD. It could be hypothesized that the gCJD patients with longer survival rates were diagnosed earlier especially because of the $43 \%$ of familial cases, and that the EEG was false negative in the earlier stage. The limited number of patients and repetitions of EEGs in the study did not allow us to differentiate the EEG pattern according to the stage of the disorder. No isolated cortical involvement was found among SCJD patients in our study as opposed to $36 \%$ of patients in Kandiah's study (16) and $41.7 \%$ in Shiga's study (17). All available MR scans (70\%) after reevaluation were CJD specific.

The main limitations of the presented study are: missing autopsy in $17 \%$ of the cases, limited genetic testing (available testing of the single PRPN mutation) and missing data in some cases. Systematic studies of EEG and MRI findings with disease progression would reveal if a lateralised finding (tricky in the early clinical phase) is merely an early sign or a part of the specific CJD phenotype.

\section{CONCLUSION}

As there are no specific therapeutic and prophylactic interventions available for prion diseases, active surveillance is critical for the control and prevention of human prion diseases. Epidemiological analysis of CJD cases in the years 2004-2016 in ES suggests that current routine surveillance systems for CJD may underestimate the true burden of disease, with special concern as regards SCJD. Awareness of the clinical signs of CJD and recognition of the different diagnostic tests available, including routine autopsy in acute/subacute dementia syndromes, creation of a database of positive tested "healthy family members" for transplantation registries and genetic counselling of affected families would be important steps for reduction of further transmission of CJD in Slovakia.

\section{Acknowledgements}

We would like to thank for the cooperation to prim. MUDr. L. Gurčík, MUDr. K. Klobučníková, PhD. and MUDr. M. Virágová.

\section{Funding}

The Operational Program for Research and Development of the Agency of the Ministry of Education of the Slovak Republic for the Structural Funds of the European Union provided financial support in the form of Project CEVA, Contract No. 034/2009/2.1/OPR\&D funding (50\%) and VEGA $1 / 0198 / 13$ a $1 / 0011 / 14$. The sponsors had no role in the design or conduct of this research.

\section{Conflict of Interests}

None declared 


\section{REFERENCES}

1. Brown P, Budka H, Cervenakova L, Collie DA, Green A, Ironside JW, et al. WHO manual for surveillance of human transmissible spongiform encephalopathies including variant Creutzfeldt-Jakob disease. Geneva: WHO; 2003.

2. Masters CL, Harris JO, Gajdusek DC, Gibbs CJ Jr, Bernoulli C, Asher DM. Creutzfeldt-Jakob disease: patterns of worldwide occurrence significance of familial and sporadic clustering. Ann Neurol. 1979;5(2):117-88.

3. Brown P, Cathala F, Gajdusek DC. Creutzfeldt-Jakob disease in France: III. Epidemiological study of 170 patients dying during the decade 1968-1977. Ann Neurol. 1979;6(5):438-46.

4. Mitrova E, Belay G. Creutzfeldt-Jakob disease with E200K mutation in Slovakia: characterization and development. Acta Virol. 2002;46(1):31-9.

5. Kovacs GG, Puopolo M, Ladogana A, Pocchiari M, Budka H, van Duijn $\mathrm{C}$, et al. Genetic prion disease: the EUROCJD experience. Hum Genet. 2005;118(2):166-74.

6. Mitrová E, Kosorinová D, Gajdoš M, Šebeková K, Tomečková I. A pilot study of a genetic CJD risk factor (E200K) in the general Slovak population. Eur J Epidemiol. 2014;29(8):595-7.

7. Mad'ar R, Maslenová D, Ranostajová K, Straka S, Baška T. Analysis of unusual accumulation of Creutzfeldt-Jakob disease cases in Orava and Liptov regions (northern Slovak focus) 1983-2000. Cent Eur J Public Health. 2003;11(1):19-22.

8. Slivarichová D, Mitrová E, Ursínyová M, Uhnáková I, Koscová S, Wsólová L. Geographic accumulation of Creutzfeldt-Jakob disease in Slovakia-environmental metal imbalance as a possible cofactor. Cent Eur J Public Health. 2011;19(3):158-64.

9. Ladogana A, Puopolo M, Croes EA, Budka H, Jarius C, Collins S, et al. Mortality from Creutzfeldt-Jakob disease and related disorders in Europe, Australia, and Canada. Neurology. 2005;64(9):1586-91.
10. Kovacs GG, Seguin J, Quadrio I, Höftberger R, Kapás I, Streichenberger N, et al. Genetic Creutzfeldt-Jakob disease associated with the E200K mutation: Characterization of a complex proteinopathy. Acta Neuropathologica. 2011;121(1):39-57.

11. Bertoni JM, Brown P, Goldfarb LG, Rubenstein R, Gajdusek DC. Familial Creutzfeldt-Jakob disease (codon 200 mutation) with supranuclear palsy. JAMA. 1992;268(17):2413-5.

12. Levy SR, Chiappa KH, Burke CJ, Young RR. Early evolution and incidence of electroencephalographic abnormalities in Creutzfeldt-Jakob disease. J Clin Neurophysiol. 1986;3(1):1-21.

13. Steinhoff BJ, Zerr I, Glatting M, Schulz-Schaeffer W, Poser S, Kretzschmar HA. Diagnostic value of periodic complexes in Creutzfeldt-Jakob disease. Ann Neurol. 2004;56(5):702-8.

14. Fulbright RK, Hoffmann C, Lee H, Pozamantir A, Chapman J, Prohovnik I. MR imaging of familial Creutzfeldt-Jakob disease: a blinded and controlled study. Am J Neuroradiol. 2008;29(9):1638-43.

15. Appel SA, Chapman J, Prohovnik I, Hoffman C, Cohen OS, Blatt I. The EEG in E200K familial CJD: relation to MRI patterns. J Neurol. 2012;259(3):491-6.

16. Kandiah N, Tan K, Pan AB, Au WL, Venketasubramanian N, Tchoyoson Lim CC, et al. Creutzfeldt-Jakob disease: which diffusion-weighted imaging abnormality is associated with periodic EEG complexes? J Neurol. 2008;255(9):1411-4.

17. Shiga Y, Miyazawa K, Sato S, Fukushima R, Shibuya S, Sato Y, et al. Diffusion-weighted MRI abnormalities as an early diagnostic marker for Creutzfeldt-Jakob disease. Neurology. 2004;63(3):443-9.

Received December 6, 2017 Accepted in revised form November 30, 2018 